Société d'histoire de la révolution de 1848 et des

révolutions du XIXe siècle

56 | 2018

Un autre $\mathrm{XIX}^{\mathrm{e}}$ siècle : I'Inde sous domination coloniale

\title{
Savoirs et colonisation en Inde : réflexions historiographiques autour des savoirs antiquaires
}

Knowledge and colonization in India: historiographical reflections based on the case of antiquarian knowledge

Wissen und Kolonisierung in Indien: Historiographische Überlegungen zu antiquarischem Wissen

Anne-Julie Etter

\section{(2) OpenEdition}

Journals

Édition électronique

URL : http://journals.openedition.org/rh19/5445

DOI : 10.4000/rh19.5445

ISSN : $1777-5329$

Éditeur

La Société de 1848

Édition imprimée

Date de publication : 15 octobre 2018

Pagination : 33-51

ISSN : 1265-1354

\section{Référence électronique}

Anne-Julie Etter, «Savoirs et colonisation en Inde : réflexions historiographiques autour des savoirs antiquaires », Revue d'histoire du XIXe siècle [En ligne], 56 | 2018, mis en ligne le 15 octobre 2020, consulté le 05 janvier 2021. URL : http://journals.openedition.org/rh19/5445 ; DOI : https://doi.org/ $10.4000 /$ rh 19.5445 


\section{ANNE-JULIE ETTER}

\section{Savoirs et colonisation en Inde : réflexions historiographiques autour des savoirs antiquaires}

Au cours du premier siècle colonial (1757-1858), appelé Company Raj, les possessions britanniques en Inde sont administrées par l'East India Company $(E I C)$. Au fur et à mesure de ses conquêtes territoriales, cette compagnie étoffe son personnel en Inde, même si le nombre d'administrateurs britanniques - percepteurs d'impôts, militaires, juges, ingénieurs et autres médecins - est toujours demeuré faible au regard de la superficie et de la démographie des territoires qu'elle contrôle. Certains d'entre eux, en parallèle de leurs obligations professionnelles, mènent des recherches sur la littérature, les sciences, les religions, l'histoire et la géographie de l'Inde. Les Asiatic Researches, revue publiée par l'Asiatic Society, créée à Calcutta en 1784 sur le modèle de la Royal Society londonienne, reflètent la variété et l'ampleur de leurs travaux. Les antiquités constituent un de leurs sujets de prédilection, bien qu'il soit quelque peu artificiel de les séparer d'autres domaines de recherche, comme l'histoire naturelle et l'astronomie, à une époque où les frontières entre les différentes branches du savoir demeurent perméables ${ }^{1}$. Ces administrateurs étudient et collectionnent des vestiges textuels et matériels : manuscrits, inscriptions, statues, monnaies, peintures, histoires orales, légendes. Ils s'intéressent particulièrement aux vestiges du brahmanisme, du bouddhisme et du jaïnisme, ainsi qu'à ceux des dynasties indo-musulmanes, dont la dynastie moghole. Ces activités favorisent le développement de savoirs antiquaires, expression utilisée pour désigner l'ensemble des connaissances liées aux vestiges du passé.

Partha Mitter a abordé ce thème par le biais de l'histoire des réactions occidentales à l'art indien. Dans Much Maligned Monsters, il soutient que la façon dont les Européens appréhendent les productions artistiques de l'Inde puise ses racines dans les récits des voyageurs qui se sont rendus dans le

1. Cf. par exemple Simon Schaffer, 'Oriental Metrology and the Politics of Antiquity in Nineteenth-Century Survey Sciences', Sciences in Context, volume 30, n 2, 2017, p. 173-212. 
sous-continent entre le XIII ${ }^{e}$ siècle et le XVII ${ }^{e}$ siècle 2 . La tendance consistant à identifier les dieux hindous à des monstres, née de la rencontre entre les traditions médiévales sur les monstres et les merveilles de l'Orient, les préjugés chrétiens des voyageurs et leur méconnaissance de l'iconographie hindoue, a notamment longtemps fait sentir ses effets, en dépit d'une connaissance peu à peu affinée de cette iconographie et de la mythologie qui lui est associée. La synthèse de Mitter demeure un outil précieux pour étudier les activités antiquaires des Européens en Inde, la constitution de collections indiennes, la réception de l'art indien en Europe, ainsi que les répercussions esthétiques, artistiques et épistémologiques de l'afflux en Europe d'objets et de connaissances sur l'art et l'architecture de l'Inde. Elle laisse toutefois de côté la question, aujourd'hui considérée comme centrale, des liens entre antiquités et colonisation.

Celle-ci conduit à s'interroger sur les conditions politiques et socio-culturelles qui ont permis aux Britanniques d'investir le champ des antiquités indiennes. Les savoirs antiquaires sont alors envisagés comme des savoirs coloniaux, ensemble disparate de connaissances qui ont en commun d'être produites en situation coloniale. Développés à la faveur de la mainmise exercée sur les territoires et les populations colonisés, les savoirs coloniaux font partie intégrante du processus de domination, comme Bernard Cohn (1928-2003) l'a montré pour l'Inde. Il évoque le cas des antiquités dans un article de 1982, republié dans Colonialism and its Forms of Knowledge: the British in India ${ }^{3}$. Les analyses de Cohn ont ouvert la voie à de nombreuses études centrées - dans le prolongement de son travail - sur le rôle des Indiens dans la constitution des savoirs coloniaux et la portée politique de ces derniers.

Bien que les liens entre savoirs et domination coloniale aient été étudiés dans d'autres contextes coloniaux, cet article traite de l'historiographie indianiste des savoirs coloniaux. À partir d'un examen des modalités de production, de diffusion et de mise en valeur des savoirs antiquaires à la fin du XVIII ${ }^{e}$ siècle et dans la première moitié du XIX ${ }^{\mathrm{e}}$ siècle, il propose une lecture critique de cette historiographie et plus particulièrement des travaux de Cohn. Je commencerai par présenter l'historiographie des savoirs coloniaux en Inde, en l'articulant autour des notions de domination et de collaboration. Ces études seront ensuite discutées en deux temps : je mettrai en relief la question des sources et des dynamiques savantes qu'elles permettent de reconstituer, avant de revenir de façon approfondie sur les liens que les savoirs antiquaires entretiennent avec l'exercice et la représentation du pouvoir.

2. Partha Mitter, Much Maligned Monsters: History of European Reactions to Indian Art, Oxford, Clarendon Press, 1977.

3. Bernard Cohn, 'The Transformation of Objects into Artifacts, Antiquities, and Art in Nineteenth-Century India', Colonialism and its Forms of Knowledge: the British in India, Princeton (N. J.), Princeton University Press, 1996, p. 76-105. 


\section{ENTRE DOMINATION ET COLLABORATION : \\ L'HISTORIOGRAPHIE DES SAVOIRS COLONIAUX EN INDE}

Soulignant les liens entre savoir et domination au sein de l'historiographie sur le colonialisme, Frederick Cooper et Ann Laura Stoler mettent en avant le travail de Cohn sur ce qui apparaît comme un régime modèle, le Raj ${ }^{4}$. Le volume consacré aux savoirs coloniaux de Rise and Fall of Modern Empires, qui propose une anthologie de textes sur divers types de savoirs dans différents empires coloniaux, reproduit quant à lui des articles de Bernard Cohn et de Christopher Bayly, crédités pour avoir mis le concept de savoir colonial sur le devant de la scène et participé au façonnement des débats sur le sujet ${ }^{5}$. Ces deux spécialistes incarnent des positions contrastées sur les savoirs dans l'Inde coloniale.

Dipesh Chakrabarty rappelle le terrain intellectuel des recherches de Cohn : succès du poststructuralisme et du postcolonialisme dans le monde anglo-saxon, poids des Subaltern Studies au sein des études indiennes et influence de l'analyse du discours colonial ${ }^{6}$. Il met en valeur le caractère pionnier de son travail qui, dès la fin des années 1960, aborde des questionnements qu'Edward Said a contribué à populariser avec son ouvrage Orientalism (1978) :

Bien avant que Foucault soit disponible pour les lecteurs anglophones ou que Said ait pris d'assaut la Bastille académique avec sa thèse rebelle sur "l'Orientalisme», Cohn [...] enseignait à ses étudiants de Chicago quelques-uns des principes de base de la relation entre savoir et pouvoir qui a façonné la carrière du colonialisme européen dans le sous-continent et ailleurs. ${ }^{7}$

Cohn, qui rattache son travail à l'œuvre de Foucault ${ }^{8}$, fait de l'interpénétration entre savoir et pouvoir "la base même du colonialisme»" Les travaux des administrateurs de l'EIC ne sont pas considérés comme une entreprise collective à l'origine d'une renaissance orientale : ils font partie du projet colonial de contrôle. En conquérant des territoires, les Britanniques ont conquis un espace épistémologique. Ils entendent organiser, classifier et

4. Frederick Cooper and Ann Laura Stoler, 'Between Metropole and Colony: Rethinking a Research Agenda', in F. Cooper and A. L. Stoler (eds), Tensions of Empire: Colonial Cultures in a Bourgeois World, Berkeley, University of California Press, 1997, p. 11.

5. Saul Dubow, 'Introduction', in S. Dubow (ed.), The Rise and Fall of Modern Empires, volume 2, Colonial Knowledges, Farnham, UK, Burlington, VT, Ashgate, 2013, p. xi.

6. Dipesh Chakrabarty, 'Foreword', in The Bernard Cohn Omnibus, New Delhi, New York, Oxford University Press, 2009, p. xiii.

7. Idem, p. x-xi.

8. Bernard Cohn, 'The Command of Language and the Language of Command', in Colonialism and its Forms..., op. cit., p. 22.

9. Ranajit Guha, 'Introduction', in Bernard Cohn, An Anthropologist Among the Historians and Other Essays, republié dans The Bernard Cohn Omnibus, op. cit. p. xviii. 
borner le monde social indien afin de pouvoir le contrôler. À cette fin, ils mettent en place des «modalités d'investigation " qui leur permettent d'identifier les informations pertinentes, puis de les organiser et de les remodeler en formes mobilisables par l'administration coloniale. Un tel processus de transformation des savoirs indiens favorise une objectification de l'Inde.

L'apprentissage des langues revêt un caractère premier. Au XVII siècle et pendant une partie du XVIII e siècle, pour mener à bien leurs opérations commerciales, les agents de l'EIC ont recours à un ensemble de détenteurs de savoirs spécialisés, qui maîtrisent les langues et les codes culturels nécessaires à la mise en place des échanges. Ils se lancent dans l'apprentissage des langues à partir des années 1740 , et plus encore après la bataille de Plassey (1757) et l'obtention de la gestion civile et fiscale du Bengale (1765). À la fin du XVIII e siècle, de plus en plus de Britanniques apprennent le persan, le sanskrit et les langues vernaculaires. Dans le même temps, un appareillage pédagogique et savant, composé de dictionnaires, de grammaires et de manuels, est produit. Les langues sont enseignées aux employés de l'EIC au Fort William College, créé à Calcutta en 1800 . Cohn voit dans ce processus l'établissement d'une formation discursive, qui participe du système de domination coloniale.

Cette analyse fait émerger la figure de l'intermédiaire. Indispensable dès le début de l'implantation commerciale des Européens, il le demeure par la suite, en raison de sa capacité à maîtriser des données dont les Britanniques ont besoin et de la faible présence numérique de ces derniers. Si la question des langues préoccupe de plus en plus les administrateurs, c'est qu'ils souhaitent mener leurs affaires commerciales et négocier avec les pouvoirs indiens sans dépendre d'interprètes, dont ils sont prompts à remettre en cause la fiabilité et l'intégrité. Ils souhaitent pour la même raison s'affranchir d'intermédiaires dans les tribunaux. Munshis et pandits demeurent pourtant incontournables, tant pour apprendre le persan et le sanskrit que pour traduire les textes de loi et les compiler en traités juridiques ${ }^{10}$. Savants, lettrés, scribes, prêtres, fonctionnaires et marchands constituent autant d'intermédiaires obligés; ils détiennent des informations qu'ils classifient et traitent pour le compte du colonisateur. Sisirkumar Das décrit le savant indien comme un "outil», un «informateur ${ }^{11}$. Pour Cohn, les Indiens sont des "sources», des "informateurs indigènes » ${ }^{12}$; ils sont convertis en instruments de domination coloniale, de même que les savoirs en leur possession ${ }^{13}$.

10. Bernard Cohn, 'Law and the Colonial State in India', in Colonialism and its Forms..., op. cit., p. $57-75$.

11. Sisirkumar Das, Sahibs and Munshis: An Account of the College of Fort William, New Delhi, Orion Publications, Calcutta, Rupa, 1978, p. 107 (cité par Bernard Cohn, 'The Command of Language...', loc. cit., p. 51).

12. Ibidem.

13. Idem, p. 21. 
Il applique cette grille de lecture à l'étude des antiquités en examinant le mouvement de collecte et de classification de données et d'objets initié pour connaître le passé de l'Inde ${ }^{14}$. Les «surveys» statistiques, opérations de relevé topographique et de collecte d'informations que l'EIC lance dans ses nouveaux territoires, ont permis de rassembler des matériaux historiques. Des objets sont acquis par le biais de pillages et d'achats; entreposés dans des musées, en Inde et en Grande-Bretagne, ils se voient conférer de nouvelles valeurs et significations, déterminées par le colonisateur ${ }^{15}$. Cohn souligne le rôle de Colin Mackenzie (1753-1821), qui met à profit ses fonctions de surveyor pour constituer une collection de plusieurs milliers d'objets (manuscrits, inscriptions, dessins, monnaies, statues, histoires orales). Il mobilise à cette fin des assistants indiens, chargés de rassembler et d'exploiter pour son compte toutes sortes de matériaux susceptibles d'éclairer l'histoire de l'Inde, à l'image de Kavali Venkata Borayya (1776-1803), interprète en chef pendant le survey du Mysore.

Les travaux de Cohn ont jeté les bases du débat sur la nature des savoirs produits dans l'Inde coloniale et le rôle des colonisés dans leur élaboration. Phillip B. Wagoner propose une synthèse des différentes thèses en présence, en distinguant deux tendances principales ${ }^{16}$. La première, qu'il rattache au courant postcolonial, comprend les travaux de Cohn, ainsi que ceux, entre autres, de Ronald Inden et de Nicholas Dirks ${ }^{17}$. Ces auteurs partagent l'idée que la supériorité militaire et économique ne suffit pas à expliquer la conquête coloniale, qui repose également sur des «technologies culturelles de domination ${ }^{18}$. Pouvoir et accumulation de savoir s'auto-entretiennent : le savoir rend possible la conquête, qui permet de mettre en place de nouveaux savoirs, qui viennent eux-mêmes consolider la conquête. Les Indiens sont passifs : ce sont des pourvoyeurs de matériaux bruts, exploités à l'aide de formes importées de savoir. La promotion de ces dernières, conjuguée à la mise à l'écart des savoirs indigènes, crée une situation de violence épistémologique.

Ce raisonnement a été largement discuté, favorisant l'émergence d'une seconde tendance, conçue comme une critique révisionniste de la vision

14. Bernard Cohn, 'The Transformation of Objects...', loc. cit.

15. Cf. aussi Richard H. Davis, Lives of Indian Images, Princeton (N. J.), Princeton University Press, 1997 , p. $143-185$.

16. Phillip B. Wagoner, 'Precolonial Intellectuals and the Production of Colonial Knowledge', Comparative Studies in Society and History, volume 45, $\mathrm{n}^{\circ}$ 4, 2003, p. 783-786.

17. Ronald Inden, Imagining India, Oxford, Basil Blackwell, 1990; Nicholas B. Dirks, Castes of Mind: Colonialism and the Making of Modern India, Princeton (N. J.), Princeton University Press, 2001. Dirks s'est intéressé de près aux travaux de Mackenzie : 'Guiltless Spoliations: Picturesque Beauty, Colonial Knowledge, and Colin Mackenzie's Survey of India', in Catherine B. Asher and Thomas R. Metcalf (eds), Perceptions of South Asia's Visual Past, New Delhi, American Institute of Indian Studies, New Delhi, Swadharma Swarajya Sangha, Madras, and Oxford \& IBH Pub. Co., 1994, p. 211-232; 'Colonial Histories and Native Informants: Biography of An Archive', in Carol A. Breckenridge and Peter Van der Veer (eds), Orientalism and the Postcolonial Predicament: Perspectives on South Asia, Philadelphia, University of Philadelphia Press, 1994, p. 279-313.

18. Nicholas B. Dirks, 'Foreword', in Bernard Cohn, Colonialism and its Forms..., op. cit., p. iv. 
postcoloniale ${ }^{19}$. Les travaux qui s'y rattachent continuent à relier les savoirs coloniaux à l'exercice du pouvoir, mais postulent qu'ils sont le fruit d'une collaboration entre colonisateurs et colonisés. Bien que les partenaires ne soient pas égaux, les colonisés participent activement à la mise en place des nouveaux savoirs, qui portent la marque de leurs catégories de pensée et régimes épistémiques. Ils les mobilisent à leurs propres fins - Cohn le souligne du reste lui-même en évoquant les effets involontaires de la formation orientaliste, que les colonisés ont pu utiliser à leur avantage ${ }^{20}$. La colonisation n'a pas provoqué de rupture épistémique : le domaine des savoirs donne à voir des continuités avec la période antérieure. Christopher Bayly a joué un rôle important dans la critique des analyses de Cohn et de Dirks, qui accordaient trop peu de crédit aux colonisés. Dans Empire and Information (1996), il montre que les Britanniques, pour pouvoir gouverner, ont dû se ménager un accès à l'ordre informationnel complexe et sophistiqué dont l'Inde était dotée. Pour maîtriser et manipuler les réseaux d'information politique, militaire et sociale, ils se sont appuyés sur des informateurs locaux, tant et si bien que «le savoir colonial a dérivé en grande partie du savoir indigène ${ }^{21}$. Bayly nuance la vision d'un pouvoir colonial omnipotent, mettant en valeur les failles et les lacunes dans la collecte et l'interprétation des informations et des connaissances.

L'idée selon laquelle des systèmes épistémologiques complexes existaient avant la conquête coloniale est partagée par Thomas R. Trautmann, qui montre que la rencontre entre diverses formes de savoir et de pensée a favorisé la création de savoirs inédits. Quelque chose de neuf, non réductible à la contribution de chacune des parties, émerge de cette confrontation. C'est le cas du concept de famille de langues dravidiennes, fruit de cadres mentaux européens et indiens : d'un côté l'ethnologie biblique et l'idée de ramification de familles de langues qui en est issue, de l'autre la discipline sanskrite d'analyse linguistique ${ }^{22}$. Les Indiens mettent dans la balance des cadres analytiques sans lesquels les nouveaux savoirs n'auraient pas émergé, ou auraient pris une forme différente. Wagoner analyse pour sa part l'émergence de l'épigraphie dans la présidence de Madras comme le résultat d'un ajustement entre l'approche cartographique et statistique du passé de Mackenzie et les compétences de ses collaborateurs, des brahmanes Niyogi attentifs à l'évolution des langues et de leur écriture et à l'utilisation potentielle de ces changements comme outils de datation. Ces compétences découlent des fonctions qu'ils occupaient au sein des cours locales avant d'intégrer l'équipe de Mackenzie ${ }^{23}$.

19. Phillip B. Wagoner, 'Precolonial Intellectuals...', loc. cit., p. 784.

20. Bernard Cohn, 'Command of Language...', loc. cit., p. 22.

21. Christopher Alan Bayly, Empire and Information. Intelligence Gathering and Social Communication in India, 1780-1870, Cambridge, New York, Cambridge University Press, 1996, p. 7.

22. Thomas R. Trautmann, Languages and Nations: the Dravidian Proof in Colonial Madras, Berkeley, University of California Press, 2006.

23. Phillip B. Wagoner, 'Precolonial Intellectuals...', loc. cit. 
Comment caractériser de tels échanges? Wagoner parle de "conversation" et de «dialogue intellectuel ${ }^{24}$, Eugene Irschick de «dialogue $»^{25}$, Trautmann de "conversation». La notion de construction dialogique modifie la façon dont le statut des acteurs indiens est appréhendé : de simples informateurs, ils deviennent des savants, des intellectuels.

Rama Mantena prend acte de l'ambivalence de ces appellations en soulignant les limites de ce que l'on pourrait appeler le modèle de la collaboration ${ }^{26}$. Il est utile selon elle de critiquer le modèle opposé, celui de la domination, qui a contribué à effacer la contribution des acteurs indigènes, alors qu'il entendait justement mettre en valeur leur marginalisation. Les Indiens sont des acteurs à part entière, mais le schéma dialogique est problématique en ce qu'il tend à gommer le rapport de force asymétrique entre les deux parties. Mantena le montre en mettant en lumière les contraintes institutionnelles et idéologiques qui pèsent sur les ambitions et les carrières des Indiens. Les Britanniques déploient à leur égard des stratégies d'inclusion et d'exclusion, qui ont pour effet de les tenir à l'écart de la communauté savante et des réseaux intellectuels. À l'instar de Ram Raz (1790-1830), auteur d'un traité sur l'architecture, et des frères Kavali, qui ont travaillé aux côtés de Mackenzie ${ }^{27}$, les Indiens peinent à se faire octroyer un statut et des postes correspondant à leurs compétences : les Britanniques les cantonnent dans une position de médiateurs, d'auxiliaires. Ce n'est que dans la deuxième moitié du XIX ${ }^{\mathrm{e}}$ siècle que des antiquaires indiens, tel Rajendralal Mitra (1823/24-1891), ont été considérés comme des savants à part entière ${ }^{28}$.

Souligner que les Indiens ont contribué de façon active à la production de nouveaux savoirs revient à faire de l'Inde coloniale un lieu d'innovations scientifiques et intellectuelles. Trautmann montre l'importance de la configuration épistémologique propre à l'Inde britannique dans le développement de la philologie historique ${ }^{29}$. Kapil Raj mobilise l'approche circulatoire des savoirs ${ }^{30}$ pour critiquer le schéma diffusionniste de la science du centre vers la périphérie : les connaissances sont co-construites par des individus et des communautés spécialisées d'Europe et d'Asie du Sud, ce qui favorise l'appa-

24. Idem, p. 811.

25. Eugene Irschick, Dialogue and History: Constructing South India, 1795-1895, Berkeley, Los Angeles, University of California Press, 1994.

26. Rama Mantena, The Origins of Modern Historiography in India: Antiquarianism and Philology, 1780-1880, New York, Palgrave Macmillan, 2012, p. 14-23.

27. Idem, p. 87-121; Rama Mantena, 'The Kavali Brothers: Intellectual Life in Early Colonial Madras', in Thomas R. Trautmann (ed.), The Madras School of Orientalism: Producing Knowledge in Colonial South India, New Delhi, Oxford University Press, 2009, p. 126-150.

28. La comparaison entre Ram Raz et Rajendralal Mitra est empruntée à Tapati Guha-Thakurta, Monuments, Objects, Histories: Institutions of Art in Colonial and Postcolonial India, New York, Chichester, Columbia University Press, 2004.

29. Thomas R. Trautmann, Aryans and British India, Berkeley and Los Angeles, University of California Press, 1997; Languages and Nations..., op. cit. Ogborn.

30. Cette approche est représentée, entre autres, par les travaux de Charles Withers et de Miles 
rition de nouvelles formes de savoir et la reconfiguration des pratiques et des savoirs existants au sein de chacune des parties; une part importante de la science occidentale ou ce que l'on considère comme telle a été produite dans les espaces extra-occidentaux ${ }^{31}$. Dans le cadre de cette «interaction constructive $»^{32}$, l'espace colonial a pu jouer un rôle de laboratoire, où de nouveaux savoirs et pratiques sont expérimentés avant d'être transférés en métropole.

\section{Les dynamiques SaVantes À L’aune des Sources}

Quels enseignements tirer de ce panorama historiographique? Il apparaît que les Indiens jouent un rôle actif dans la production des savoirs, bien que la domination coloniale fasse sentir ses effets d'un bout à l'autre de la chaîne savante. Deux traits, eux-mêmes liés, se dégagent également d'emblée : la portée des savoirs antiquaires dans la réflexion sur les savoirs coloniaux et la primauté de la figure de Mackenzie dans cette historiographie. Ce constat, on va le voir, met en relief la problématique des sources, bien connue des spécialistes d'histoire coloniale amenés à composer avec la version tronquée et partiale du fait colonial qu'offrent les documents émis par le colonisateur. Les sources dont on dispose pour étudier les savoirs antiquaires mettent en valeur à la fois le rôle des Indiens et l'effacement dont il a fait l'objet. Il est alors intéressant d'analyser les modalités et les motifs de cet effacement.

Les travaux de Cohn, de Dirks, de Wagoner ou encore de Mantena proposent des interprétations divergentes de l'œuvre de Mackenzie, mais ont ceci en commun de lui accorder une place importante. Cela tient tout d'abord à l'ampleur du projet du surveyor et au dispositif qu'il a déployé pour le mener à bien. Mackenzie se donne pour objectif d'étudier et d'écrire l'histoire de l'Inde à l'aide de matériaux collectés à travers le sous-continent ${ }^{33}$. La tâche est immense. Pour l'accomplir, il s'appuie sur un réseau de correspondants européens et met sur pied une équipe d'assistants, européens et indiens. Il recourt à des collaborateurs indiens, car il ne connaît aucune langue locale; ceux-ci maîtrisent en outre les réseaux nécessaires à l'identification et à la collecte de documents et d'objets. Mackenzie leur envoyait des instructions détaillées et des questionnaires destinés à guider leur travail; ses assistants rédigeaient des rapports faisant état de la progression de leurs investigations, à l'image du Report of Baboo Rao étudié par Cohn ${ }^{34}$. Mackenzie est aussi l'auteur de nombreux mémoires et rapports adressés aux autorités de l'EIC. Dans ces

31. Kapil Raj, Relocating Modern Science: Circulation and the Construction of Scientific Knowledge in South Asia and Europe: Seventeenth to Nineteenth Centuries, Delhi, Permanent Black, 2006, p. 223.

32. Kapil Raj, "Hégémonie impériale ou interaction constructive? L'Inde coloniale au XIX siècle", in Kapil Raj et H. Otto Sibum [dir.], Histoire des sciences et des savoirs, volume 2, Modernité et globalisation, Paris, Le Seuil, 2015, p. 305-325.

33. Jennifer Howes, Illustrating India: the Early Colonial Investigations of Colin Mackenzie (1784-1821), New Delhi, New York, Oxford University Press, 2010.

34. Bernard Cohn, 'The Transformation of Objects...', loc. cit., p. 84-85. 
Fig. 1. Portrait de Colin Mackenzie avec trois pandits indiens par Thomas Hickey (1816).

๑ $\odot$ British Library Board, Foster 13.

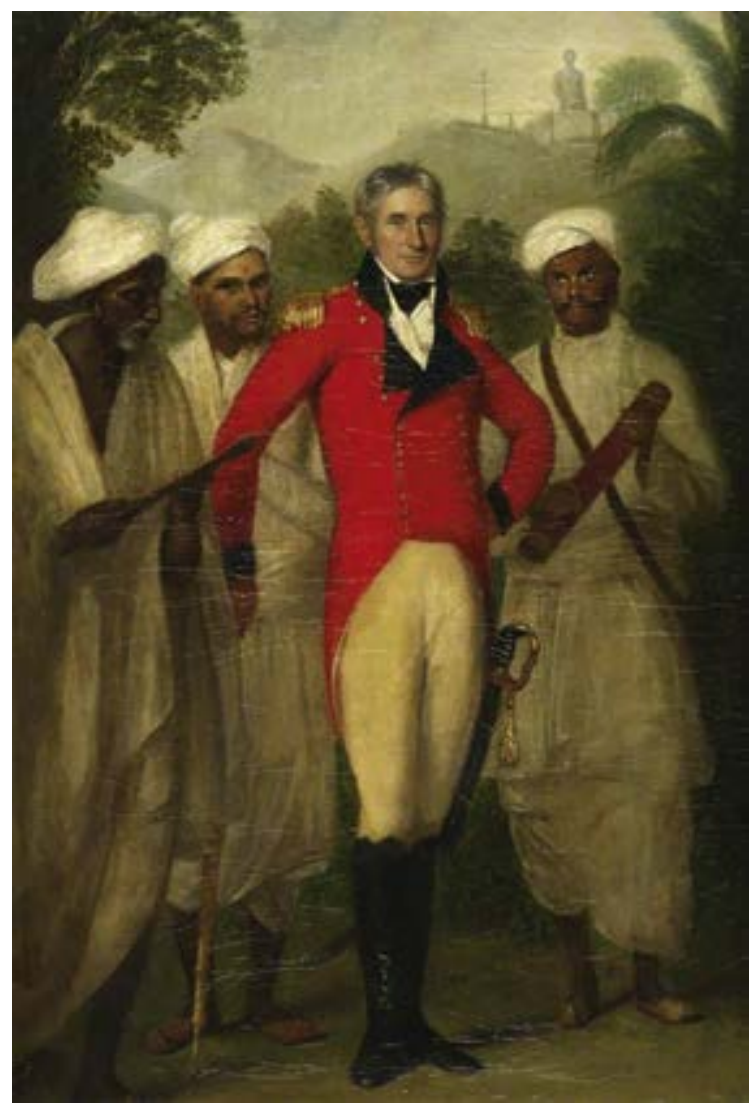

documents, il fait état de la composition de son équipe et donne des détails sur ses assistants indiens : nom, informations biographiques, salaire, tâches effectuées. Attentif à faire valoir l'importance de leurs travaux, il se montre préoccupé de leur sort. Au moment de la clôture du survey du Mysore, puis en 1815-1816, alors qu'il s'apprête à quitter Madras pour Calcutta, il défend ainsi le droit de ses assistants à des pensions et se préoccupe de leur devenir. En 1816, il propose que cinq d'entre eux soient transférés au collège de Fort Saint-George, où il compte déposer une centaine de manuscrits, afin qu'ils puissent travailler sur ces matériaux ${ }^{35}$. Une telle sollicitude le distingue du reste des administrateurs ${ }^{36}$. Un tableau de Thomas Hickey (1816), presque invariablement convoqué dès lors qu'il est question de Mackenzie, illustre les rapports qu'il entretenait avec ses assistants (fig. 1). L'antiquaire est représenté entouré de trois pandits qui tiennent un télescope et un manuscrit, une statue à l'arrière-plan signalant son intérêt pour la religion jaïne. Un dernier

35. Lettre de Mackenzie au gouvernement de Fort Saint George, $1^{\text {er }}$ août 1816 (National Archives of India, Survey of India, Reports, $N^{\circ} 3$, p. 74).

36. Nicholas B. Dirks, 'Colin Mackenzie: Autobiography of an Archive', in Thomas R. Trautmann (ed.), The Madras School..., op. cit., p. 41. 
point a son importance : de multiples documents émanant de sa main ont été conservés, de même que le vaste ensemble de matériaux qu'il a collectés ou fait collecter, regroupés sous le nom de "collection Mackenzie». Le cas de Mackenzie peut ainsi, à plusieurs égards, être qualifié d'exceptionnel. Ce trait, rarement interrogé sous un angle historiographique, est significatif: les caractéristiques de l'œuvre de Mackenzie et l'existence de documents permettant de l'étudier contribuent à expliquer l'importance des réflexions sur les savoirs coloniaux dans les débuts de l'Inde britannique et la forme qu'elles ont prises, mettant l'accent à la fois sur la figure de l'intermédiaire et le domaine des antiquités.

Les collaborateurs de Mackenzie ne sont pas les seuls à être identifiés. On connait les pandits de William Jones, figure de proue de l'orientalisme britannique : Radhakanta Tarkavagisha et Sarvoru Candra Tripathi, tous deux employés dans les cours de justice britanniques, ou encore Ramlochan ${ }^{37}$. Sankaraiah et Vedam Pattabhirama Shastri faisaient quant à eux partie du cercle de Francis Whyte Ellis, au nom duquel est attachée la "preuve dravidienne» étudiée par Trautmann, bien qu'elle soit l'œuvre collective d'Indiens et d'Européens. En 1816, le Board of Revenue demande à Ellis, alors collecteur de Madras, de fournir une liste détaillée des employés indigènes de son administration, au sein de laquelle Sankaraiah occupait la fonction de chef du personnel. Ce document permet de se faire une idée de sa carrière. Cet exemple est singulier : il est rare que les Indiens apparaissent dans les archives coloniales ou que celles-ci permettent de retracer leur biographie ${ }^{38}$. Les Indiens sur lesquels nous disposons d'informations sont avant tout ceux qui étaient au service de l'EIC ou qui, pour une raison ou une autre, ont été en contact avec l'administration coloniale. Ils ne forment toutefois qu'une partie des acteurs qui ont participé au développement de nouvelles connaissances. D'autres intervenants figurent dans les articles, les ouvrages et les comptes rendus de visites de monuments des administrateurs, tels les desservants d'édifices religieux, qui leur rapportent l'histoire et les légendes du lieu et leur montrent des inscriptions, ou encore des érudits et des fonctionnaires locaux.

Qu'il existe ou non des sources permettant d'identifier des acteurs indiens et d'étudier leur parcours, il demeure malaisé d'évaluer leur contribution. En publiant une traduction des inscriptions gravées sur le pilier d'Asoka Delhi-Topra, Jones précise qu'elles ont été traduites du sanskrit, «ainsi qu'elles ont été expliquées " ${ }^{39}$ par Radhakanta. Il rapporte avoir bénéficié de l'aide de pandits pour identifier des noms propres et interpréter certaines

37. Cf. par exemple Rosane Rocher, 'The Career of Rādhākānta Tarkavāgiśs, an Eighteenth-Century Pandit in British Employ', Journal of the American Oriental Society, volume 109, $\mathrm{n}^{\circ} 4,1989$, p. 627-633.

38. Thomas R. Trautmann, Languages and Nations..., op. cit., p. 103-104.

39. William Jones, 'Inscriptions on the Staff of Firuz Shah. Translated From the Sanscrit, As Explained by Ra'dha'ca’nta Sarma', Asiatick Researches, 1, 1788, p. 379-382. 
expressions. Leur apport s'est-il vraiment limité à la description qu'en donne Jones? Rien ne permet de l'affirmer. À l'image de cet exemple, on peine à cerner le rôle effectif des Indiens, car on ne dispose la plupart du temps que du témoignage qu'en ont laissé les antiquaires britanniques. Or, ceux-ci tendent à minimiser l'apport de leurs interlocuteurs indigènes, réduit à une contribution d'appoint. Les textes des administrateurs fourmillent de pandits, de brahmanes, de munshis et de fakirs anonymes, au sujet desquels on ne sait rien si ce n'est qu'ils ont "aidé" à traduire des textes et des inscriptions ou à interpréter la statuaire. Les acteurs indigènes disparaissent parfois complètement. Les Britanniques s'approprient des échanges et des connaissances, dont ils s'attribuent la paternité. S'il est difficile, en l'état de la documentation, de restituer l'apport respectif des uns et des autres, il est possible de mettre au jour les ressorts de ce mécanisme de captation.

Mohamad Tavakoli-Targhi montre que Jones, « directeur d'une entreprise savante d'envergure $»^{40}$, s'est approprié le produit du capital intellectuel et du travail des pandits et des savants persanophones de son réseau. La célébrité de Jones, considéré comme l'un des fondateurs de la grammaire comparée et de la linguistique historique, repose sur une hypothèse qu'il formule en 1786 : les affinités entre le sanskrit, le grec et le latin sont telles qu'il est raisonnable de conclure que ces langues sont issues d'une source commune, peut-être disparue. Il rattache le gothique, le celte et le vieux persan à cet ensemble de langues, aujourd'hui connu sous le nom de langues indo-européennes ${ }^{41}$. Le caractère pionnier du travail de Jones est à nuancer. Dans la préface de sa grammaire bengalie (1778), Nathaniel Halhed souligne une similitude entre mots sanskrits, latins et grecs, tandis que le jésuite Courdoux, en 1767, envoie à l'Académie des inscriptions et belles-lettres une liste de termes sanskrits illustrant leur parenté avec d'autres langues anciennes et modernes. Le lexicographe et linguiste Siraj al-Din Khan Arzu (1689-1756) avait quant à lui montré les affinités entre le persan et le sanskrit. Jones ne mentionne pas les travaux d'Arzu, mais des preuves textuelles permettent d'affirmer qu'il s'en est servi. Ils font partie de ce que Tavakoli-Targhi appelle les textes oubliés de l'orientalisme. Jones et d'autres administrateurs se sont arrogés des travaux en persan, qu'ils avaient pour certains commandés : ils les ont publiés en anglais sous leur nom, se contentant parfois d'ajouter le dispositif académique de notes de bas de page et de références. Ce phénomène d'usurpation peut être mis en relation avec la dislocation du système de patronage savant en Inde et l'émergence de nouveaux principes d'attribution textuelle en Europe. En traduisant et en publiant ces textes, les orientalistes ont effacé la trace de leurs producteurs indigènes. Pour rétablir la place de ces derniers dans l'histoire

40. Mohamad Tavakoli-Targhi, 'The Homeless Texts of Persianate Modernity', in Ramin Jahanbegloo (ed.), Iran Between Tradition and Modernity, Lanham (Md.), Oxford, Lexington Books, 2004, p. 142.

41. William Jones, 'The Third Anniversary Discourse, Delivered 2d February 1786. By the President', Asiatick Researches, 1, 1788, p. 422-423. 
de l'orientalisme, il convient d'explorer les archives de textes persans non publiés et de restituer les relations intertextuelles entre les travaux des orientalistes et «leurs textes originaux orientaux qui ont été réprimés» ${ }^{42}$.

Le cas des textes persans étudié par Tavakoli-Targhi signale le processus éditorial comme une étape importante de l'effacement de la contribution indigène. L'exemple de Mackenzie, qui se préoccupait bien plus de collecte que de publication ${ }^{43}$, l'illustre également. Sa collection présente l'intérêt d'offrir un accès à la contribution brute des membres de son équipe. Le titre des documents est significatif, comme "Account of the Ruins \& Sculptures at Mahavellyporam by Cavelly Venkata Boria Bramin Sent by Capt. Mackenzie to Examine Them in December 1799 ${ }^{44}$. De telles sources permettent d'étudier la contribution de Borayya ${ }^{45}$ et son œuvre d'historien-ethnographe ${ }^{46}$. Un des rares articles de Mackenzie, publié en 1807 dans les Asiatic Researches, est intitulé "Account of the Jains. Collected from a Priest of This Sect at Mudgeri: Translated by Cavelly Boria, Brahmen, for Major C. Mackenzie». La participation de Borayya, qui a organisé le compte rendu à partir de son travail de terrain et l'a enrichi d'extraits de textes visant à expliciter les croyances et les cérémonies décrites, est réduite à une opération de traduction. Borayya tire ses informations d'un "prêtre» au sujet duquel on ne sait pas grand-chose, dupliquant en quelque sorte le processus d'effacement des contributeurs le long de la chaîne de production intellectuelle. Bien que Mackenzie reconnaisse par ailleurs les compétences de son assistant, c'est son nom, non celui de Borayya - encore moins celui des interlocuteurs de ce dernier -, qui est attaché à ce mémoire, considéré comme un travail pionnier sur le jaïnisme.

La publication est bel et bien un moment clé de l'oblitération de la contribution des Indiens. Le processus d'appropriation sous-jacent est une manifestation, parmi d'autres, de la mainmise exercée par les Britanniques sur la société colonisée. Les aspirations savantes des administrateurs jouent également à plein. Alors que les Indiens peinent à faire reconnaître leurs compétences, ils ont l'opportunité de développer des carrières savantes. Une résidence plus ou moins prolongée en Inde en tant qu'employé civil ou militaire de l'EIC offre des débouchés professionnels et des possibilités d'enrichissement; elle est aussi l'occasion d'acquérir une expertise sur les antiquités indiennes, que les administrateurs coloniaux cherchent notamment à faire valider auprès des institutions et des réseaux métropolitains. Pour ce faire, ils mobilisent leurs collections : les objets dont ils font don à des sociétés

42. Mohamad Tavakoli-Targhi, 'The Homeless Texts...', loc. cit., p. 145.

43. Lisa Mitchell, 'Knowing the Deccan: Enquiries, Points, and Poets in the Construction of Knowledge and Power in Early-Nineteenth-Century Southern India', in Thomas R. Trautmann (ed.), The Madras School..., op. cit., p. 154-155.

44. British Library, Oriental and India Office Collections, Mss Eur Mack Gen 21, p. 281-286.

45. Nicholas B. Dirks, 'Colin Mackenzie...', loc. cit., p. 39-40.

46. Rama Mantena, The Origins of Modern..., op. cit., p. 99-104. 
savantes ou à des musées métropolitains incarnent leur légitimité savante, acquise localement ${ }^{47}$. Ils mettent également en avant la familiarité qu'ils entretiennent avec leur objet d'étude, faisant valoir qu'ils maîtrisent une ou plusieurs langue(s) indienne(s) et qu'ils ont une connaissance de première main des vestiges de l'Inde - ils ont par exemple visité les monuments et les sites qu'ils décrivent et dont ils retracent l'histoire. De façon similaire, leurs échanges avec des savants ou des lettrés indiens peuvent représenter une forme de caution scientifique. C'est la raison pour laquelle ils prennent soin, dans les articles et les ouvrages qu'ils publient, de mentionner qu'ils ont été en contact avec des pandits et autres munshis, tout en s'y référant de façon elliptique et en minimisant leur participation : le souhait de certains administrateurs de se forger une réputation savante éclaire le traitement qu'ils réservent à leurs interlocuteurs indiens dans leurs travaux.

\section{ANTIQUités, ADMINisTRATION COLONIALE ET POUVOIR : UN FAISCEAU DE RELATIONS}

Prendre en compte les intérêts et les trajectoires des administrateurs vient aussi enrichir l'examen des liens entre les savoirs et le fonctionnement de l'administration coloniale. Cohn affirme, à propos de la modalité d'investigation appelée muséographique, que le pouvoir de définir la nature du passé et d'établir des priorités dans la création d'une archive monumentale d'une civilisation représente un instrument majeur de souverainetée $e^{48}$. On peut admettre avec lui que les collections d'objets anciens et les découvertes archéologiques ont permis, au même titre que les gazettes et les recensements, d'englober l'Inde et de mieux diriger sa population. Il ne détaille cependant guère les ressorts de ce processus. Pour cela, il est possible de se référer à ce qu'il avance au sujet de la "modalité historiographique», séparant la construction idéologique de la nature de la civilisation indienne, qui vient légitimer le pouvoir colonial, des enquêtes de type historique liées à une nécessité administrative, comme la collecte de coutumes et d'histoires locales que les Britanniques rattachent aux régimes fonciers et au calcul de l'impôt ${ }^{49}$. Cohn n'exploite pas cette distinction de façon systématique; elle constitue cependant une grille de lecture utile.

Les savoirs antiquaires représentent un outil idéologique efficace, car ils sont un moyen de cantonner les colonisés dans une position inférieure.

47. Anne-Julie Etter, 'Collecting Statues in India and Transferring Them in Britain or the Intertwined Lives of Indian Objects and Colonial Administrators (Late $18^{\text {th }}$ Century-Early $19^{\text {th }}$ Century)', in Claire Gallien and Ladan Niayesh (eds), Eastern Resonances, à paraître. Cette analyse fait écho au concept de self-fashioning, qui signale que les collectionneurs utilisent leurs collections pour se façonner de nouvelles identités sociales et culturelles (Maya Jasanoff, Edge of Empire: Conquest and Collecting in the East, 1750-1850, London, Fourth Estate, 2005).

48. Bernard Cohn, Colonialism and its Forms..., op. cit., p. 10.

49. Idem, p. 5-6. 
L'Inde, a-historique et statique, est placée du côté de la tradition et du mythe, à l'opposé du progrès et de la rationalité incarnés par la Grande-Bretagne. Les colonisateurs, qui affirment qu'ils apportent à l'Inde les méthodes de l'investigation historique, se donnent pour tâche d'écrire une histoire de l'Inde, pour l'Inde : les catégories privilégiées - déclin, chaos, corruption, stagnation - viennent légitimer leur établissement sur le sol indien. Cela s'accompagne d'une dévalorisation des compétences des Indiens, qui seraient dépourvus de sens historique et d'esprit critique. Les capacités d'observation nécessaires à l'examen des monuments leur font défaut : seuls les Britanniques seraient capables de "voir» correctement sur le terrain et donc de produire des comptes rendus fiables ${ }^{50}$. S'ils ont des compétences antiquaires, ils les ont acquis au contact des colonisateurs : en 1850, John Wilson, président de la Bombay Asiatic Society, valorise ainsi les recherches de Vishnu Sastri Bapat, tout en précisant que c'est parce que les Britanniques ont montré aux Indiens la valeur historique des inscriptions qu'il a pu développer des talents épigraphiques ${ }^{51}$.

La catégorie du déclin politique est appliquée aux monuments, qui en seraient le reflet. Maintenir ces vestiges en bon état participe alors du mouvement de régénération que les Britanniques prétendent apporter à l'Inde. Tout au long du premier siècle colonial, ils financent des travaux d'entretien et de réparation d'édifices anciens, privilégiant les temples et les mosquées en activité et les vestiges moghols, comme le Taj Mahal et le mausolée d'Akbar à Sikandra (fig. 2). L'EIC entend capter un peu du prestige et de la légitimité attachés à la dynastie moghole, tout en se pliant au devoir de patronage des institutions religieuses dévolu au souverain indien. La maintenance des édifices relève bien de l'exercice et de la légitimation du pouvoir. Pour autant, elle n'entre pas en écho avec la documentation antiquaire produite à l'initiative des administrateurs : une grande partie des édifices auxquels ils consacrent articles et ouvrages sont des vestiges brahmaniques, jaïns et bouddhiques à l'abandon. Les mesures d'entretien et de réparation produisent des connaissances, puisqu'elles nécessitent, en amont, un travail de compilation d'informations sur le plan, la décoration et l'histoire des édifices qui implique administrateurs et acteurs indigènes, tels les gardiens ou les desservants du site. Ce travail, dont le fruit est conservé dans les archives de l'EIC, se développe en parallèle des recherches antiquaires menées à titre individuel par les administrateurs, mais ne les recoupe $\mathrm{pas}^{52}$. Différents types

50. Sudeshna Guha, Artefacts of History. Archaeology, Historiography and Indian Pasts, Los Angeles, London, New Delhi, SAGE Publications, 2015, p. 38-41.

51. Idem, p. 103.

52. Anne-Julie Etter, 'Antiquarian Knowledge and Preservation of Indian Monuments at the Beginning of the Nineteenth Century', in Daud Ali and Indra Sengupta (eds), Knowledge Production, Pedagogy, and Institutions in Colonial India, Basingstoke, Palgrave Macmillan, 2011, p. 75-95; «La conservation des monuments en Inde. Variations à partir du Company Raj (fin du XVIII siècleannées 1850)», Cahiers du CAP, volume 3, 2016, p. 57-76. 


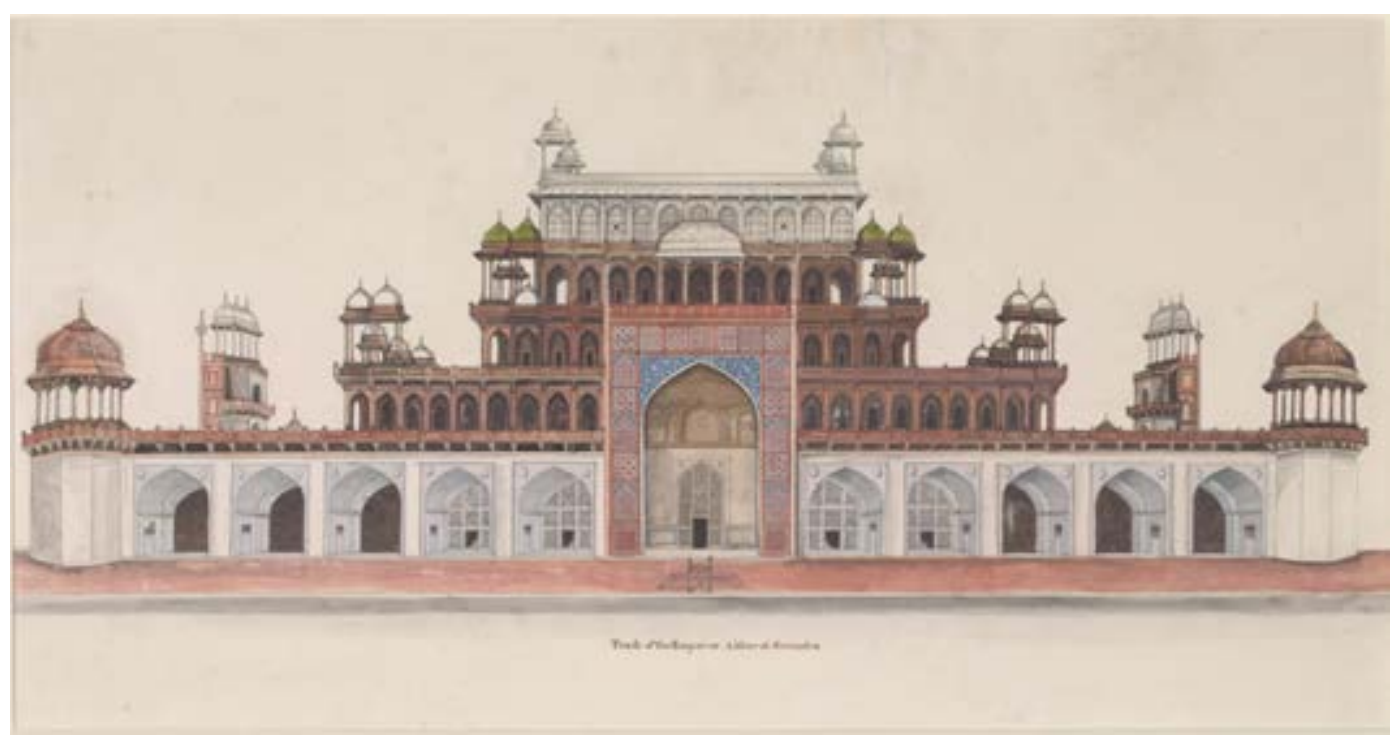

Fig. 2. Tombe de l'empereur Akbar à Sikandra (Fifteen drawings of Mughal architecture and ornamental detail on Mughal monuments at Agra, vers 1816-1822).

(C) Victoria and Albert Museum, London, IM 187-1920.

et outils de connaissances sur les monuments, destinés à des usages et à des publics variés, sont mis en place.

Les recherches épigraphiques offrent un exemple d'exploitation des savoirs antiquaires dans une perspective administrative. Elles sont convoquées dans le cadre des interrogations sur la nature des régimes agraires et fiscaux à adopter. La raison en est que le corpus épigraphique consiste principalement en donations de terres et de villages à des institutions ou à des personnalités religieuses, offrant un éclairage sur les questions de propriété. F. W. Ellis, dans son Treatise of Mirasi Rights (1818), oriente l'étude des inscriptions vers la question de l'existence ou non d'une forte propriété terrienne en Inde du $\mathrm{Sud}^{53}$. Mackenzie signale lui aussi l'intérêt des inscriptions pour les réflexions sur les baux agricoles et l'évaluation de l'impôt. Plus généralement, il promeut l'étude des antiquités, soulignant «l'utilité de cette entreprise pour le gouvernement actuel $\aleph^{54}$. Dans sa correspondance officielle, il ne cesse d'affirmer que les matériaux historiques peuvent guider l'EIC dans la gestion de ses possessions indiennes. Cela tient en partie au fait que ses recherches antiquaires, qu'il mène de son propre chef, ne répondent à aucune directive. En insistant sur leur portée administrative, il se livre à une entreprise de communication et de promotion destinée à convaincre ses supérieurs de l'intérêt

53. Thomas R. Trautmann, 'Riot Over Ryotwar', in T. R. Trautmann (ed.), The Madras School..., op. cit., p. 310-322.

54. Lettre au secrétaire du gouverneur général à Calcutta (25 juin 1805). National Archives of India, Survey of India, Memoirs, No. 18, p. 122. 
de son travail et à susciter leur concours, sous forme de mesures destinées à le faciliter ou d'un appui financier. De ce point de vue, il a obtenu gain de cause. Le destin de sa collection, délaissée par le pouvoir colonial qui la considérait comme un ensemble de documents fantaisistes et peu fiables, indique toutefois qu'elle n'a pas eu le statut d'outil administratif qu'il proposait de lui conférer. De même, Richard Johnson, désireux de convaincre les directeurs de l'EIC d'acheter sa collection (1807), insiste sur ses potentiels avantages administratifs. Il vante l'intérêt de ses manuscrits, qui pourront servir au collège de Haileybury, nouvellement créé pour former le personnel civil envoyé en Inde, et de ses "traités statistiques très rares et précieux sur les recettes " 55 . Quant aux miniatures, elles favoriseraient une meilleure connaissance des territoires contrôlés par l'EIC.

Ces exemples d'amplification de l'utilité politique et administrative de l'érudition orientaliste et des collections d'antiquités amènent à distinguer les usages administratifs effectifs de ce qui relève d'un souci de publicité ou de promotion d'un projet particulier ou individuel ${ }^{56}$. On met alors l'accent sur les stratégies personnelles des administrateurs, en les distinguant du positionnement des instances dirigeantes de l'EIC, qui encouragent les recherches antiquaires pour autant qu'elles les aident, à peu de frais, à asseoir leur pouvoir. De leur côté, les Indiens essaient de négocier leur participation, espérant obtenir quelque avantage en retour. Les discussions portent souvent sur les dotations des institutions religieuses et l'entretien des bâtiments. Des membres de la communauté jaïne acceptent de montrer des inscriptions à l'équipe de Mackenzie, mais réclament en échange une intervention auprès des autorités de l'EIC afin d'obtenir des fonds qui leur permettraient de réparer leurs temples et de célébrer leurs cérémonies. Dans son histoire de Jaunpur (1802), traduite en anglais en 1814, Khair ud din Muhamad Ilahabadi décrit les monuments de la ville et offre la traduction d'une inscription trouvée dans le fort, espérant mobiliser le gouvernement colonial en faveur d'une réparation des édifices ${ }^{57}$.

Le regard vers le passé imprègne les principes de gouvernance mis en place par l'EIC. Une sorte d'orientalisme officiel est promu dans les années 1770-1780, dans le but de conférer de la respectabilité au gouvernement du Bengale ${ }^{58}$. Les Britanniques prétendent gouverner l'Inde en accord avec ses institutions et ses traditions, ce qui a pu être analysé comme un

55. Lettre à C. Wilkins (30 janvier 1807), citée par Mildred Archer and Toby Falk, Indian Miniatures in the India Office Library, London, Sotheby Parke Bernet, Delhi, Karachi, Oxford University Press, 1981, p. 26.

56. Pour une analyse similaire du projet orientaliste de Warren Hastings, Rosane Rocher, 'British Orientalism in the Eighteenth Century: the Dialectics of Knowledge and Government', in Carol Breckenridge and Peter van der Veer (eds.), Orientalism and the Postcolonial..., op. cit., p. 215-249.

57. Sur Jaunpur, Michael S. Dodson, 'Jaunpur, Ruination, and Conservation During the Colonial Era', in Daud Ali and Indra Sengupta (eds.), Knowledge Production..., op. cit., p. 123-146.

58. Christopher A. Bayly, Empire and Information..., op. cit., p. 52. 
processus de restauration constitutionnelle, censé justifier leur action ${ }^{59}$. Le recours aux savoirs antiquaires en vue de l'élaboration de politiques administratives a cependant été marginal. Cela ne veut pas dire qu'il n'existe pas de liens entre le développement de l'administration coloniale et l'étude et la conservation des vestiges du passé; bien au contraire, ils sont multiples. Cette relation se voit aux fonctions professionnelles des acteurs impliqués, aux modalités concrètes de collecte et de circulation des objets et des données, à la dimension idéologique de l'étude des antiquités ou encore à sa portée administrative, notamment par le biais du patronage ${ }^{60}$. Les savoirs antiquaires ne forment pas pour autant un bloc uniforme : composés de strates diverses, ils sont susceptibles de donner lieu à des applications et à des appropriations variées. Une grande hétérogénéité caractérise les savoirs dans l'Inde coloniale, à de multiples niveaux : contenu, conditions de production, cohérence interne, alignement avec les politiques administratives, concrétisations institutionnelles, relations avec le pouvoir colonial, modalités de réception ${ }^{61}$. Les savoirs coloniaux se caractérisent de façon générale par leur nature dynamique, fragmentée et contingente, en dépit des relations de pouvoir dans lesquelles ils s'inscrivent ${ }^{62}$.

Les savoirs antiquaires offrent un angle d'attaque intéressant pour explorer les savoirs coloniaux dans l'Inde britannique, en raison de la place notable qu'ils occupent au sein de l'historiographie consacrée à ce sujet. Un élément d'explication réside dans la spécificité de l'œuvre de Mackenzie et l'existence de sources permettant d'étudier les rapports qu'il entretenait avec ses interlocuteurs indiens, véritable point aveugle dans la plupart des cas. Les travaux de Cohn ont contribué à poser les jalons de ces questionnements. Force est de constater qu'ils ont conservé une grande actualité, malgré leurs limites. Comme le souligne Dipesh Chakrabarty, ils alimentent encore la réflexion, pour peu que l'on envisage le colonialisme comme macro-phénomène ${ }^{63}$. Cohn a le mérite d'avoir mis en relief les liens entre savoir et domination coloniale, tout en offrant des outils pour les étudier. Son analyse n'en appelle pas moins une réévaluation et un approfondissement, que ce soit du point de vue des interactions entre Indiens et Britan-

59. Robert Travers, Ideology and Empire in Eighteenth-Century India: the British in Bengal, Cambridge, Cambridge University Press, 2007.

60. Anne-Julie Etter, Les Antiquités de l'Inde: monuments, collections et administration coloniale (1750-1835), Thèse d'histoire sous la direction de Marie-Noëlle Bourguet, Université Paris Diderot, 2012, $511 \mathrm{f}^{\circ}$.

61. Daud Ali and Indra Sengupta, 'Introduction', in D. Ali and I. Sengupta (eds), Knowledge Production..., op. cit., p. 11.

62. Par exemple, Ricardo Roque and Kim A. Wagner (eds), Engaging Colonial Knowledge: Reading European Archives in World History, Basingstoke, New York, Palgrave Macmillan, 2012.

63. Dipesh Chakrabarty, 'Foreword', loc. cit., p. xvi. 
niques ou des relations concrètes que la production des savoirs entretient avec le déploiement et le fonctionnement de l'administration coloniale.

Ce travail de relecture critique, déjà mené par de nombreux chercheurs, peut être complété sur certains points. Alors que le schéma de l'informateur passif ne tient plus guère la route, je partage l'idée d'une participation active des Indiens à la formation des savoirs, sans pour autant adopter la notion de collaboration ou de dialogue, dès lors qu' elle relègue à l'arrière-plan la profonde inégalité qui marque ces échanges. Aujourd'hui encore demeure une hésitation sur le statut à accorder aux Indiens, ce que reflètent les appellations utilisées : savant, intellectuel, informateur, intermédiaire, assistant, collaborateur. La restitution du rôle des acteurs indigènes se limite aux quelques cas pour lesquels on dispose de sources; elle ne peut être qu'incomplète et en partie conjecturale. Il est en revanche possible d'approfondir l'analyse du processus d'effacement dont ils ont fait l'objet, en s'interrogeant sur ses ressorts et ses effets. J'ai montré que cette oblitération passait notamment par la transcription, sous forme de publications, des savoirs dont les Indiens sont les détenteurs, étape elle-même centrale dans les stratégies savantes des administrateurs britanniques. Pour ce qui est des liens avec l'administration coloniale, le schéma de la domination demeure pour l'essentiel valide. Il demande néanmoins à être affiné pour que ces liens puissent être appréhendés dans toute leur diversité et leur complexité, en s'interrogeant par exemple sur la portée idéologique des catégories de pensée associées aux antiquités et la façon dont les connaissances antiquaires ont été traduites en politiques administratives ou mobilisées à des fins personnelles par les administrateurs. Les savoirs antiquaires mêlent une grande variété d'acteurs et d'intérêts, ce qui rend problématique toute interprétation monolithique. Il convient plutôt de mettre au jour les motivations et les trajectoires des administrateurs, en distinguant ces derniers de l'administration coloniale en tant qu'institution, ainsi que celles des Indiens, malgré les restrictions qui pèsent sur leurs actions et leurs carrières.

Les travaux de Cohn, enfin, sous-tendent une approche en termes de rupture qui mérite d'être revue dès lors que l'on estime que les connaissances qui se développent au cours de la période coloniale portent la marque des régimes épistémiques indigènes. Les pratiques et les savoirs antiquaires en Inde dans les siècles antérieurs à la colonisation font l'objet d'un intérêt croissant ${ }^{64}$, dans le cadre d'une analyse comparée de l'antiquariat à l'échelle mondiale ${ }^{65}$. Leur étude peut entrer en résonance avec celle

64. Sudeshna Guha, Artefacts of History..., op. cit., p. 30-66; Sanjay Subrahmanyam, 'Traces of the Ancients in India: Notes on Two Possible Narratives', in Alain Schnapp (ed.), World Antiquarianism: Comparative Perspectives, Los Angeles, Getty Research Institute, 2014, p. 372-385.

65. Peter N. Miller and François Louis (eds), Antiquarianism and Intellectual Life in Europe and China, 1500-1800, Ann Arbor (Mich.), University of Michigan Press, 2012; Alain Schnapp (ed.), World Antiquarianism..., op. cit. 
des savoirs antiquaires à l'époque coloniale, dans la mesure où elle permet de mieux appréhender les continuités et les changements à l'œuvre à la fin du XVIII ${ }^{e}$ siècle et au XIX ${ }^{\mathrm{e}}$ siècle.

Anne-Julie Etter est maîtresse de conférences en histoire contemporaine à l'Université de Cergy-Pontoise 